\b

Volumen $6 \mathrm{~N} .{ }^{\circ} 45$

julio - diciembre de 2018

ISSN: 0122-4328

ISSN-E: 2619-6069

pp. $66-73$

\title{
Juventud rural e identidad: entre desarraigos $y$ resistencias
}

\author{
Rural Youth and \\ Identity: Between \\ Uprooting and \\ Resistance
}

\author{
Juventude \\ camponesa: entre \\ desenraizamento e \\ resistência
}

Karen Lorena Jiménez Guevara*

Fecha de recepción: 20-10-18

Fecha de aprobación: 30-10-18

PARA CITAR ESTE ARTÍCULO

Jiménez, K. (2018). Juventud rural e identidad: entre desarraigos y resistencias. Nodos y nudos, 6(45), 66-73. doi: 10.17227/nyn.vol6.num45-8321

\footnotetext{
* Magister en Educación, licenciada en Educación Básica con énfasis en Ciencias Sociales. Actualmente es docente en Universidad Santo Tomás. krenjg0808@gmail.com
} 


\section{RESUMEN}

El presente artículo visualiza los resultados de un trabajo de investigación, adelantado en el Municipio de la Jagua de Ibirico, en el Departamento del Cesar, con un grupo de campesinos caficultores, víctimas del conflicto armado en la zona y que, en la actualidad se encuentran en proceso de recuperación de tierras. El trabajo realizado con los campesinos, permitió analizar categorías como subjetivación, educación, resistencia y desarraigo, reflejando la situación de los jóvenes, los cuales se encuentran expuestos a diversas situaciones, que los llevan a tomar decisiones de abandonar sus tierras o de adelantar procesos de resistencia a favor de la defensa de su territorio. Por consiguiente, la investigación se desarrolló bajo una metodología cualitativa- hermenéutica, lo que posibilitó la participación y reflexión de los caficultores, en torno a temas como la educación, expulsión de sus tierras, entorno de los jóvenes y las pocas oportunidades en la zona rural.

Palabras clave: educación rural; desarrollo; jóvenes rurales; ruralidad; territorio

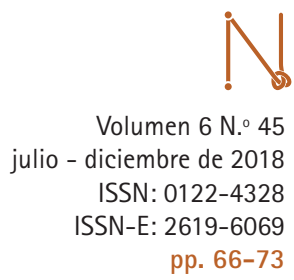

pp. 66-73

\section{ABSTRACT}

This article shows the results of a research project carried out in the municipality of La Jagua de Ibirico, in the Department of Cesar, with a group of coffee farmers, victims of the armed conflict in the area, who are currently in the process of land recovery. The work done with the peasants allowed to analyze categories such as subjectivation, education, resistance and uprooting, reflecting the situation of young people, who are exposed to different situations, which lead them to make decisions to leave their lands or to advance processes of resistance in favor of the defense of its territory. Therefore, the research was developed under a qualitative-hermeneutic methodology, which allowed the participation and reflection of coffee farmers, around issues such as education, expulsion from their land, young individuals environment and few opportunities in rural áreas.

Key words: rural education; development; rural youth; rurality; territory

\section{RESUMO}

Este artigo mostra os resultados de um projeto de pesquisa, realizado no município de La Jagua de Ibirico, no Departamento de César, com um grupo de cafeicultores vítimas do conflito armado na área, atualmente no processo de recuperação de terras. 0 trabalho realizado com os camponeses permitiu analisar categorias como subjetivação, educação, resistência e desenraizamento, refletindo a situação dos jovens, expostos a diferentes situações, que os levam a tomar decisões para sair de suas terras ou avançar processos de resistência em favor da defesa de seu território. Portanto, a pesquisa foi desenvolvida sob uma metodologia qualitativa-hermenêutica, que permitiu a participação e reflexão dos cafeicultores, em torno de questões como educação, expulsão de suas terras, meio ambiente de jovens e poucas oportunidades em áreas rurais.

Palavras-chave: educação rural; desenvolvimento; juventude rural; ruralidade; território 
Las zonas rurales han estado expuestas al acaparamiento de tierras, a la producción de agrocombustibles y al monocultivo, justificados en el sostenimiento de una forma de vivir especifica, reflejada en las ideas de éxito, felicidad y progreso, instauradas desde la lógica mercantil global. Indirectamente, esta obliga a los campesinos a consumir productos, semillas, químicos, tecnología y otros elementos, muchas veces innecesarios pero que hacen parte de la calidad de vida y el bienestar, y que son instaurados por el imaginario occidental capitalista que pretende que todas las comunidades sigan un camino ya trazado de progreso y consumo direccionado desde la modernidad y la necesidad de instaurar lógicas económicas en todas las dimensiones del ser humano.

De esta manera, la idea de desarrollo permite determinar cuáles son los países desarrollados y subdesarroIlados, creando una jerarquización política, económica, cultural y social, donde las grandes potencias generan directrices a seguir para los demás países. Estos, por su carácter de subdesarrollo, deben seguir al pie de la letra, a fin de crear las condiciones necesarias para reproducir en todo el mundo los rasgos propios de las sociedades avanzadas, caracterizadas por la industria, la urbanización, altos niveles de vida y el uso de la tecnología como insumo para la instauración de la modernidad en todas las esferas sociales.

Por ende, y como lo menciona Lang y Mokrani (2011, p. 27)

El desarrollo es un dispositivo de poder que reorganizó el mundo, relegitimando la división internacional del trabajo en contexto capitalista, mediante un enorme conjunto de discursos y prácticas, convirtiéndose en el objetivo de las políticas públicas, destinando presupuestos e instituciones encargadas de impulsar dicha idea de desarrollo.

Por consiguiente la idea de desarrollo instaurada en las zonas rurales ha ocasionado minimización cultural, desarraigo, transferencia de tecnología y desconocimiento del saber campesino, en correspondencia con las nuevas formas de vida, las nuevas lógicas mercantiles y la idea de progreso como sinónimo de calidad de vida, bienestar y adelanto económico. Esto causa un impacto en la parte educativa, social y cultural de las zonas rurales, a través de mecanismos de control instaurados por las sociedades avanzadas y desarrolladas.

El discurso de desarrollo desde la parte educativa, se refleja en la necesidad de impartir conocimientos especializados en la zona rural, enfatizando en labores y oficios que aporten al desarrollo económico de la región, vinculando de forma sistemática conocimientos y prácticas (formas de conocer y de poder) por medio de proyectos, intervenciones particulares y programas educativos informales, a fin de insertar al joven campesino en las lógicas del mercado global y en correspondencia con los modelos económicos, culturales y políticos de las potencias mundiales.

En ese sentido, se empieza a reflejar la necesidad de encasillar a los jóvenes en programas especializados que respondan a la lógica económica imperante, permitiéndoles a las grandes empresas o multinacionales cualificar mano de obra, obteniendo obreros más formados y especializados con la misma remuneración e incluyéndolos en el sistema educativo, como mecanismo directo tanto de control, como de capacitación relacionada con mano de obra, industrialización del campo e idea de desarrollo.

La educación impuesta desde las necesidades de los organismos internacionales no es más que una serie de requerimientos y estándares que ven en la academia una plataforma informacional que capacita a los usuarios de productos y servicios para que optimicen el sistema de compra y venta sin cuestionar los altos índices de desigualdad y las percepciones de infelicidad y pobreza que se experimentan a diario. (Castro, 2017, p. 16).

Esto nos permite hacer relación directa con los programas de educación, implementados en la zona rural y como muy pocos de ellos tienen en cuenta el joven adulto; el cual, representa un porcentaje significativo en la tasa de analfabetismo de la zona rural; $y$ otros implementan programas que no tienen nada que ver con las caracteristicas del contexto ni el sistema de producción de las zonas rurales; provocando que los jóvenes dejen el sistema educativo formal y se adhieran rápidamente al sistema económico como obreros y jornaleros; muchas veces sin saber leer ni escribir. 
Dicha situación genera retención de jóvenes en la minería, en la construcción y la extracción de hidrocarburos, con remuneración económica baja y pocas condiciones laborales, ya que ello representa tener una estabilidad económica, que les permitirá estar a la par con los jóvenes que viven en la ciudad, los cuales, poseen bienes materiales, representando de esta manera, calidad de vida y progreso. Por consiguiente, la mayoría de jóvenes abandonan los procesos educativos formales, dejando de lado la idea de profesionalizarse, ya que el mismo "desarrollo económico" les recalca que lo único que necesitan es saber un oficio para poderse emplear y ganar dinero. ¿Es esto justo con los jóvenes rurales, a quienes se les limita la educación y la profesionalización.

Los jóvenes del Cesar tienen pocas posibilidades. Por ejemplo, acá en Jaguas, no hay colegios, solo escuelas, entonces los que quieran ir a estudiar su bachillerato deben dirigirse a Valledupar y para eso se necesita plata, plata que muchos no tienen, entonces los jóvenes prefieren aprender a manejar moto e irse por ahi. Sin embargo, el SENA sí nos llega hasta acá, pero son cursos en relación con la mina, a manejar volquetas y demás cosas del trabajo de la mina, situación que nos dañó mucho el comercio y la producción agrícola en el municipio. (Caficultor del departamento del Cesar, 2017).

La educación de jóvenes y adultos ha sido una problemática abordada en menor medida, pero que ha sido foco de la idea de desarrollo y sus intereses económicos en la zonas rurales, ya que los jóvenes y adultos representan una población altamente productiva que requiere de intervención educativa, para la transferencia de conocimiento acorde con las dinámicas de la economía global, convirtiéndolos rápidamente en obreros, impidiendo así, que los jóvenes construyan su proyecto de vida relacionado con las características culturales, económicas y sociales de su familia y territorio.

De esta manera, se evidencian las pocas oportunidades que poseen los jóvenes rurales, quienes por terminar sus estudios, conseguir mejores ingresos, son expulsados de su tierra, siguiendo la idea de progreso y desarrollo instaurada en la zona rural, donde invita a que los jóvenes abandonen sus tierras, su familia y su identidad para mejorar su calidad de vida; pero alguien ha preguntado, ¿cuál sería la calidad de vida para ellos? Preguntas que no existen en el actual modelo educativo rural.

Los jóvenes que no son expulsados pasan a ser mano de obra en contextos mineros, camioneros, volqueteros, distanciándolos cada vez más de sus raíces y de lo que significa vivir en el campo; por consiguiente, se observa cómo la educación de jóvenes es un mecanismo de control económico, donde se busca la homogenización de prácticas, modos y formas de vivir en el campo, obligando directa o indirectamente a transformar sus estilos de vida, sus cultivos y sus identidades por pertenecer económicamente a un estilo de vida.

¡No se trata solo de que los jóvenes tengan que quedarse obligadamente en el campo! Por supuesto que deben tener la posibilidad de elegir: "la alternativa de la residencia urbana es un derecho de toda persona" (Durston, 1998, p. 114). Pero el objetivo debe ser que tengan un abanico real de opciones entre las que puedan elegir. Es decir, entre otras cosas, que el joven debe alcanzar una educación y formación que le permitan un desarrollo profesional/personal tanto en el espacio rural como en el urbano. Para alcanzar esto -que no se logra actualmente - está claro que debe haber políticas específicas, que tengan en cuenta los numerosos y variados factores que influyen en ese proceso de decisión. ¿Nacer en el campo-morir en la ciudad?

En razón a lo anterior, es necesario resaltar que en las zonas rurales se consolida una nueva generación de jóvenes que construyen una experiencia territorial caracterizada, asemejada y diferenciada de otras generaciones por tener mayor movilidad e intercambio con otros entornos (cabeceras municipales, centros urbanos donde acceden a bienes y servicios), lo que posibilita el intercambio de saberes en entornos geográficos diferentes.

De igual forma, son jóvenes que poseen mayores niveles educativos que sus padres y abuelos, lo que conlleva a que tengan expectativas mayores en la educación superior como proyecto de vida; sin embargo, existen barreras sociales, económicas y geográficas de acceso y permanencia en el sistema educativo, dado que no hay ciudad por pequeña que sea, que no 
imponga al mundo rural, sus limitaciones, falta de posibilidades y muchas veces desconocimiento de los habitantes rurales, como sujetos políticos.

Los jóvenes que habitan el campo manifiestan diversas aspiraciones académicas; sin embargo, la oferta académica en la zona rural no responde a las expectativas de dichos jóvenes, dado que el abanico de opciones está orientado en su mayoría a la producción agropecuaria, a través del SENA, desconociendo que las zonas rurales necesitan médicos, maestros, ingenieros, etc., para el fortalecimiento de sus costumbres y la preservación de los recursos naturales.

Asimismo, dichos jóvenes poseen habilidades e intereses en las nuevas tecnologias de la comunicación y la información, permitiendo que tengan acceso a las comodidades del mercado, a los servicios mercantiles y financieros de manera más fácil.

Esta nueva generación construye un sentido y un vínculo con su territorio, más allá de la producción de alimentos, lo que refleja una sensibilidad frente a las amenazas que se ciernen sobre el medio ambiente, donde muchos de estos jóvenes lideran procesos en defensa de su tierra y sus recursos. El trabajo agrícola para muchos de estos jóvenes, es pesado, mal remunerado y se encuentra por fuera de sus expectativas, sumado a esto, existen muchas dificultades para que ellos puedan acceder a tierras.

Estos jóvenes experimentan situaciones no resueltas como el acceso a la salud y a la educación, una acelerada degradación al medio ambiente, las profundas brechas y desigualdades que existen entre lo urbano y lo rural, sumado a que han crecido en entornos con múltiples y permanentes violencias derivadas del conflicto armado. De igual forma, la función social de la ciudad genera que de alguna forma se acrecenten dichas situaciones, dado que en su seno residen los centros institucionales del poder, desde donde se organiza y estructura la sociedad.

En muchas ocasiones, la percepción que estos jóvenes tienen del campo es mediada tanto por los discursos construidos en la ciudad sobre el mundo rural, como por las situaciones que han vivido, como la guerra, el desplazamiento forzado, la pérdida de familiares, el menosprecio por ser habitante rural en múltiples espacios y la falta de oportunidades. Las mencionadas condiciones estructurales fortalecen estas miradas y los jóvenes se sienten en desventaja frente a sus pares urbanos, por la falta de oportunidades educativas y económicas. No obstante, algunos jóvenes expulsados del campo a la ciudad manifiestan un cierto desencanto por la ciudad, generado por su experiencia en la ciudad, dado que la promesa de "vivir bien la ciudad" se desvanece, cuando estos jóvenes deben luchar con las dinámicas urbanas, reflejadas en inseguridad, falta de solidaridad, comidas, aumento de los costos de vida y demás variables que caracterizan ser habitante de la ciudad.

\section{Algunas consideraciones finales}

Si bien es cierto, desde la organización estatal, existen proyectos que buscan desarraigar y expulsar a los jóvenes de su territorio, simultáneamente en las comunidades rurales, se adelantan propuestas para fortalecer el arraigo y la defensa de su territorio, donde los jóvenes son participantes en organizaciones y procesos colectivos de resistencia, fomentando la autonomía, el diálogo de saberes, la defensa de la vida y el territorio, resignificando la mirada de lo rural. Dichos procesos de resistencia generan prácticas organizativas que conllevan a construir relatos, experiencias, propuestas y vínculos entre las comunidades en defensa de los saberes, recursos, costumbres y resignificación de lo que significa crecer y vivir en el campo.

Por otro lado, la migración de los jóvenes rurales hacía poblados vecinos o grandes ciudades está motivada por la búsqueda de oportunidades educativas y laborales, dada la poca oferta de condiciones materiales y sociales para permanecer en el campo. En este sentido, la migración rural no puede leerse del todo como desarraigo, o como necesidad de los jóvenes por desvincularse o distanciarse de sus comunidades, tal como lo menciona Osorio, Jaramillo y Orjuela (2011, p. 38): 
Se puede salir de manera forzada o voluntaria, no retornar $y$, pese a ello, querer y valorar su territorio. Salir, partir, no necesariamente significa rechazo, desapego u olvido del territorio. Las migraciones internas, con diferente grado de coacción y de libertad, en las cuales las y los jóvenes ocupan un lugar relevante, constituyen una fuente silenciosa en inadvertida de recomposición de la sociedad colombiana.

En Colombia, existe un desconocimiento de quiénes son esos jóvenes rurales, sus necesidades, sus expectativas, sus sueños, reproduciendo percepciones construidas desde la ciudad, donde en muchas ocasiones se piensa que son un simple obrero, sin carácter político, crítico ni propositivo, reflejando cierta superioridad de los jóvenes urbanos sobre sus pares, los jóvenes rurales, respondiendo a tres tendencias sobre las juventudes rurales: a) la invisibilización de dichos jóvenes en otros contextos, b) la poca oferta de servicios educativos y económicos, c) la homogenización de los habitantes de la zona rural, desconociendo la diversidad de problemáticas, necesidades, caracteristicas, costumbres y dinámicas propias de su territorio.

Lo anterior conlleva al desconocimiento tanto del joven rural, como del territorio en su totalidad, generando propuestas que poco o nada tienen que ver con lo que de verdad necesita la población que habita el campo, pensando que los proyectos o propuestas implementadas en lo urbano, darán los mismos resultados en campesinos, indígenas o afrodescendientes, invisibilizando las prácticas de formación propias, de resistencia y defensa de su territorio.

\section{Referencias}

Castro, A. (2017). La participación política en la escuela como alternativa a la cultura del silencio. Nodos y Nudos, 5(43), 13-20. Recuperado de http://revistas.pedagogica.edu.co/ index.php/NYN/article/view/8520

Díaz, G., Ortiz, P., y Núñez, I. (2004). Interculturalidad, saberes campesinos y educación. Tlaxcala: El colegio de Talxcala, SEFOA, Fundación H. Böll.

Durston, J. (1998). Juventud y Desarrollo Rural: marco conceptual y contextual. Santiago de Chile: Cepal.

Escobar, A. (1999). Antropología y desarrollo. Maguaré, 14, 42-73.

Escobar, A. (2002). Globalización, desarrollo y modernidad. En Corporación Región (Ed.), Planeación, Participación y Desarrollo (pp. 9-32). Medellin: Corporación Región.

Escobar, A. (1998). La invención del Tercer Mundo: construcción y deconstrucción del desarrollo. Bogotá: Editorial Norma.

Escobar, A. (2005). El "postdesarrollo" como concepto y práctica social. En D. Mato (Coord.), Políticas de economía, ambiente y sociedad en tiempos de globalización (pp. 1731). Caracas: Facultad de Ciencias Económicas y Sociales, Universidad Central de Venezuela.

Escobar, A., Álvarez, S. y Dagnino, E. (2001). Introducción. Lo cultural y lo político en los movimientos sociales latinoamericanos. En A. Escobar, S. Álvarez y E. Dagnino (Eds.), Politica cultural y cultura política. Una nueva mirada sobre los movimientos sociales latinoamericanos (pp. 1748). Bogotá: icanh, Taurus.

Lang, M. y Mokrani, D. (Orgs.) (2011). Más allá del desarrollo. Quito: Fundación Rosa Luxemburg; AbyaYala.

Osorio, F. E., Jaramillo, O. y Orjuela, A. (2011). Jóvenes rurales: identidades y territorialidades contradictorias. Algunas reflexiones desde la realidad colombiana. Énfasis. Boletín del Observatorio Javeriano de Juventud, (1), 1-40. 


\section{Diálogo del conocimiento}

El artículo pone en el centro de su análisis, la migración de los jóvenes rurales a contextos urbanos, en búsqueda del progreso y el bienestar instaurados desde el modelo de desarrollo. El discurso del desarrollo ha traido desarraigo y desconocimiento de los saberes campesinos, y ha impactado todos los aspectos de la vida en las zonas rurales; por supuesto, la educación no está por fuera de este alcance. Se plantea entonces que el discurso propio del desarrollo ha traido la reducción de la educación al aprendizaje de saberes que contribuyan al crecimiento económico, la cualificación de mano de obra o la industrialización del territorio.

Plantea también el documento que la educación en las zonas rurales se convierte en un mecanismo de control económico desde el que se dictamina, por ejemplo, qué debe entenderse por bienestar. Aunque en el texto se hace una crítica a cómo la educación es instrumentalizada a favor del modelo de desarrollo instaurado, se demanda a la vez la presencia de posibilidades para que los jóvenes rurales alcancen niveles altos de profesionalización dentro del sistema educativo instaurado y se habla de cerrar brechas entre lo rural y lo urbano.

Es fundamental la emergencia de discusiones alrededor de la relación entre desarrollo y educación, y la problematización del tipo de educación que se instaura en las zonas rurales. Quizá, avanzar en esta construcción implique superar conceptos como el de brecha (que finalmente termina poniendo a lo urbano como un fin alcanzable), y ahondar en la posibilidad de construir modelos de educación propia desde los territorios, que se distancien del sistema educativo convencional, o que por lo menos dialoguen de otras formas con este. Pensar en cuestionar el modelo de desarrollo implica reconocer modos de ser y estar en los territorios, y por supuesto de construir para qués propios alrededor de asuntos como el bienestar o la educación. 